\title{
Roland Chollet, L'CEuvre de Balzac en préfaces. Des romans de jeunesse au théâtre,
}

\section{Patrick Berthier}

\section{(2) OpenEdition}

1 Journals

\section{Édition électronique}

URL : http://journals.openedition.org/studifrancesi/1387

DOI : 10.4000/studifrancesi. 1387

ISSN : 2427-5856

Éditeur

Rosenberg \& Sellier

\section{Édition imprimée}

Date de publication : 1 décembre 2015

Pagination : 607-609

ISSN : 0039-2944

\section{Référence électronique}

Patrick Berthier, « Roland Chollet, L'EFuvre de Balzac en préfaces. Des romans de jeunesse au théâtre, », Studi Francesi [En ligne], 177 (LIX | III) | 2015, mis en ligne le 01 décembre 2015, consulté le 06 janvier 2021. URL : http://journals.openedition.org/studifrancesi/1387 ; DOI : https://doi.org/10.4000/ studifrancesi. 1387

Ce document a été généré automatiquement le 6 janvier 2021.

\section{(c)}

Studi Francesi è distribuita con Licenza Creative Commons Attribuzione - Non commerciale - Non opere derivate 4.0 Internazionale. 


\section{Roland Chollet, L'Euvre de Balzac en préfaces. Des romans de jeunesse au théâtre,}

Patrick Berthier

\section{RÉFÉRENCE}

ROLAND CHOLLET, L'CEuvre de Balzac en préfaces. Des romans de jeunesse au théâtre, textes réunis par Marie-Bénédicte DIETHELM et Nicole MOZET, Paris, Classiques Garnier, 2014, pp. 536.

1 La disparition de Roland Chollet, balzacien incomparable qui nous a tant donné, fait de ce magnifique volume préparé avant sa mort un hommage funèbre; mais ce n'est pas un livre triste, puisque par cette publication le commentateur éclairé qu'il fut demeure, au contraire, étonnamment vivant parmi nous, aujourd'hui, dans la modernité de sa vision de Balzac. Voilà en effet plus de cinquante ans (entre 1958 et 1962) que les textes de ce volume ont été écrits; or, les références inévitablement datées mises à part, ils sonnent comme s'ils étaient vraiment nés d'hier, par-delà toute évolution pédante ou non de la critique. Voici pourquoi.

2 Lorsqu'il a rédigé pour les éditions Rencontre, à Lausanne, des préfaces pour chacun des trente volumes d'une nouvelle édition des Euvres complètes de Balzac, Roland Chollet a voulu proposer un travail vraiment nouveau. Voisinaient alors sur le marché, achevées ou en cours, plusieurs grandes éditions: celle de Marcel Bouteron et Henri Longnon chez Conard (quarante volumes de 1912 à 1940); ce que les balzaciens appellent entre eux «vieille Pléiade», édition assurée par Bouteron seul en dix volumes de cette collection de 1935 à 1937, et complétée en 1959 par un onzième tome, confié à Roger Pierrot lui aussi récemment disparu; l'édition dirigée par Albert Béguin et JeanA. Ducourneau pour Formes et Reflets, 16 volumes publiés de 1950 à 1953 et repris de 1962 à 1964 par le Club français du livre; enfin l'édition Maurice Bardèche au Club de 
l'Honnête Homme en vingt-huit volumes parus de 1955 à 1963, et qui est donc la seule à ne pas être terminée lorsque Roland Chollet se met au travail. La Pléiade et Bardèche proposent La Comédie humaine dans l'ordre voulu par Balzac et fixé, pour l'essentiel, par le catalogue dressé par lui en 1844 (pour l'essentiel, car quelques redistributions d'une section à l'autre de l'œuvre sont encore décidées après cette date). Béguin et Ducourneau, en revanche, ont adopté «un ordre nouveau» déterminé par la date de l'intrigue de chaque roman, en toute conscience des problèmes que posent à cet égard les romans à large empan chronologique. Roland Chollet prend un autre risque, non moins réel: celui de présenter les œuvres en suivant la date non de leur intrigue, mais de leur composition; pour les romans à plusieurs épisodes, la date choisie sera celle de l'achèvement, soit 1843 pour Illusions perdues ou 1847 pour Splendeurs et misères des courtisanes.

3 Indépendamment des discussions qu'il peut susciter, un tel choix a évidemment pour avantage de faire relire Balzac dans l'ordre de l'élaboration de l'œuvre, tout en proposant une forme originale de biographie intellectuelle. Pour rendre cet aspect plus sensible, les éditrices - c'est une de leurs rares interventions de quelque importance ont déplacé les préfaces des premiers romans (de 1822 à 1825) en tête du volume, alors que chez Rencontre les œuvres de jeunesse étaient reléguées vers la fin de l'ensemble; seuls se trouvent toujours à la fin la préface des Contes drolatiques inachevés (publiés de 1833 à 1837), ainsi que ce qui concerne le théâtre, réuni en un vaste chapitre allant de Cromwell (1819-1820) au Faiseur, créé après la mort de Balzac dans une version défigurée. Nous nous trouvons finalement face à trois groupes de préfaces, celles des premiers romans (pp. 13-118), celles des romans de La Comédie humaine (pp. 123-425), enfin celles des Contes drolatiques et du théâtre (pp. 427-501); le volume se termine sur une postface de Marie-Bénédicte Diethelm consacrée à une évocation qui nous en apprend beaucoup sur les travaux de Roland Chollet, mais dont le ton dithyrambique l'aurait fait sourire, modeste inguérissable qu'il était.

4 Ce qui est vrai, en revanche, c'est que la lecture de ces cinq cents pages de préfaces mises bout à bout produit un effet sidérant sur l'esprit du lecteur qui croyait bien connaître son Balzac; lue ou plutôt évoquée dans ce nouvel ordre, La Comédie humaine révèle des trésors et suscite sans cesse des envies de relecture. La distribution des romans dans chaque volume se faisant en fonction non seulement de leur date d'écriture mais de leur longueur, on voit se côtoyer textes brefs et textes longs, scènes parisiennes et de province, œuvres achevées ou non. On avance avec le plaisir de redécouvrir, et dans le désir de redécouvrir encore.

5 À coup sûr ce fut une belle idée éditoriale que de vouloir faire un livre de la succession de ces préfaces inspirées. Roland Chollet préfère à l'exhaustivité, impossible dans l'espace dont il dispose, un éclairage, mais qu'il pousse à fond: ici sur la genèse, là sur la technique des personnages reparaissants qui crée peu à peu un «roman sans frontières» (p. 331), ailleurs encore sur des thèmes fondamentaux de la vision sociale et morale de Balzac, et partout sur la structure générale et particulière de l'œuvre entière. On se dit sans cesse avec étonnement que tout cela a été écrit avant la mode du structuralisme, avant l'invasion de la narratologie et de pas mal d'autres spécialités technicisantes: car si Roland Chollet fut dès cette époque, notamment dans ce cycle de préfaces, un des plus grands généticiens de l'œuvre de Balzac, c'est bien en n'oubliant jamais la littérature. Pas de dissection, mais de vivants conseils pour lire en toute 
intelligence une œuvre certes inachevée mais qui, «malgré ses voûtes éventrées et ses niches vides, [...] donne une impression de plénitude et de perfection» (p. 125).

On ne sait où puiser tant la visite du monument est sans cesse passionnante, que Roland Chollet s'attache à sortir de l'ombre un élément méconnu comme Les Petites Misères de la vie conjugale (pp. 152-156) ou qu'en deux pages denses il fasse le point sur la question mouvante du «romantisme» de Balzac (pp.171-172). Les remarques lumineuses ne manquent pas non plus sur le rôle du lecteur, et sur «notre conscience, avec laquelle l'écrivain joue et hors de laquelle l'œuvre ne peut vivre» (p. 302): Roland Chollet parle souvent de la mémoire de celui qui lit Balzac et qui ne peut bien lire Balzac que s'il fait, d'un roman à l'autre, travailler cette mémoire grâce à laquelle il percevra ce que le critique appelle dans plusieurs des préfaces l'unité de destinée des personnages, reparaissants ou non. Des années après avoir écrit tous ces textes, Roland Chollet, qui se trouvait alors en pleine rédaction de sa superbe thèse Balzac journaliste (Klincksieck, 1983), a été chargé par Pierre-Georges Castex, directeur de la nouvelle édition «Pléiade» publiée de 1976 à 1980, de réaliser l'édition d'Illusions perdues, et les balzaciens savent à quel point cette édition est insurpassable; or on constate que la préface de ce même roman pour Rencontre, quinze ans plus tôt (dans notre volume pp.331-342), était déjà extraordinairement riche malgré sa brièveté, ne serait-ce que sur le thème de la «délégation» évoqué dans les mêmes années par Gaëtan Picon dans son excellent Balzac par lui-même (Seuil, 1956), petit ouvrage trop négligé aujourd'hui et auquel on s'étonne un peu que Chollet ne renvoie pas.

7 Un mot sur la réalisation matérielle du volume, au cas où l'on m'aurait trouvé trop dityhrambique sur le fond (mais saurait-on l'être!). Je me suis demandé pourquoi, au fil des pages, on trouvait tant de coquilles non corrigées, y compris portant sur des noms propres connus - ainsi, à quelques lignes de distance, p. 493, sur l'acteur Lockroy (imprimé «Locroy») et sur le directeur des théâtres de la banlieue, Seveste (imprimé "Sévestre»); d'autres gênent la lecture, fût-ce en faisant sourire, comme lorsque Sarrasine est "détrompée» (sic, au féminin, p. 169) sur l'identité du castrat qu'il a pris pour une femme... Sans doute ces fautes-là viennent-elles de coquilles non aperçues; mais j'ai fini par me convaincre que la plupart, parfois de taille, proviennent de ce que, pour réaliser la présente édition, on a scanné les textes des années soixante: technique ô combien précieuse par les gains de temps qu'elle permet, mais dont on connaît les ravages en l'absence d'un contrôle scrupuleux des résultats. Quand on lit, p. 229, «bien que Vautrin ne sait rien», on saisit aussitôt qu'il faut lire «soit»... mais ce n'est pas au lecteur d'assumer cette correction. Même chose pour les avatars de la conjonction «mais», qui devient «riais» p. 239 et «niais» p. 370, en produisant ici et là, bien sûr, une phrase absurde. D'autres résultats aberrants comme "quelle» au lieu de «qu'elle» (p. 106), «soif l'histoire» (au lieu de «soit», p. 324) ou «celle envergure» (pour «cette», p. 332) ont évidemment pour origine la même absence de contrôle après le scannage. Si l'on ajoute à ces défauts quelques coquilles sur les titres («Illusion perdues», p. 340, ou «La Tour de Nesles», p. 468), on se retrouve tout de même face à un bilan qui n'est pas celui qu'il devrait être, surtout ici où il s'agissait de rendre à un chercheur aimé l'hommage qu'il mérite, donc un hommage lui-même irréprochable. Roland chollet était évidemment le premier à savoir que le titre du drame de Dumas ne prend pas d's, mais même si la faute était dans la page d'origine des éditions Rencontre, elle ne devait pas subsister chez Garnier. 
8 Il n'était pas question de dissimuler cette ombre au tableau, car elle était sur bien des points évitable; mais il ne faut pas non plus lui donner trop d'importance, car ce qui doit compter avant tout, dans le bonheur de lecture que nous procure ce grand livre, c'est le fait que son auteur ne l'avait pas conçu comme tel, puisque bien sûr chaque préface était isolée en tête de chaque volume, mais que la réunion de toutes les préfaces produit réellement l'œuvre d'un maitre, un peu comme la réunion des romans de $L a$ Comédie humaine produit un roman unique, le livre de Balzac. Et tant pis si la modestie de Roland Chollet, déjà évoquée plus haut, souffre de ce rapprochement qui, à moi, me saute aux yeux. 